\title{
Fabrication and demonstration of an electrochromic voxel array for a volume display prototype
}

\author{
Seiichi Takamatsu ${ }^{\text {a) }}$, Sachio Murao, Nguyen Binh-Khiem, \\ Eiji Iwase, Kiyoshi Matsumoto, and Isao Shimoyama \\ Graduate School of Information Science and Technology, the University of Tokyo \\ 7-3-1 Bunkyo-ku, Hongo, Tokyo 113-8656, Japan \\ a)seiichi-takamatsu@aist.go.jp
}

\begin{abstract}
We fabricated and demonstrated electrochromic voxel arrays for a three-dimensional (3D) display prototype. A voxel is a cubic display element of a 3D image. The voxels were constructed with ultraviolet-curable polymer on a substrate with a MEMS process and coated with poly-(3,4-ethylenedioxythiophene):poly-styrenesulfonate (PEDOT:PSS). The resultant substrates were stacked in three-dimensional space. The PEDOT:PSS color changes from transparent to blue when $-1 \mathrm{~V}$ is applied. The coloration of one voxel was observed from the top and side views, and the coloration of a $3 \times 3 \times 3$ voxel array was also observed.
\end{abstract}

Keywords: volume display, electrochromism, PEDOT:PSS

Classification: Micro- or nano-electromechanical systems

\section{References}

[1] J. Son, B. Havidi, and K. Kwack, "Methods for displaying threedimensional images," Proc. IEEE, vol. 94, pp. 502-523, 2006.

[2] T. E. Clifton and F. L. Wefer, "Direct volume display devices," IEEE Comput. Graph. Appl., pp. 57-65, 2006.

[3] G. E. Favalora, "Volumetric 3D Displays and Application Infrastructure," Computer, vol. 38, no. 8, pp. 37-44, 2005.

[4] J. P. Boeuf, "Plasma display panels: physics, recent developments and key issues," J. Phys. D, vol. 36, R53-R79, 2003.

[5] A. Argun, P. Aubert, B. Thomson, I. Shwendeman, C. Gaupp, J. Hwang, N. Pinto, D. Tanner, A. MacDiamid, and J. Reynolds, "Multicolored electrochromism in polymers: structures and devices," Chemistry of Materials, vol. 16, pp. 4401-4412, 2004.

[6] H. Kimura, T. Uchiyama, and H. Yoshikawa, "Laser produced 3D display in the air," Proc. SIGGRAPH 2006, Emerging technologies 20, 2006.

[7] A. Chekhovski and H. Toshiyohi, "3-dimensional water display," IEICE Electron. Express, vol. 4, pp. 430-434, 2007. 


\section{Introduction}

Recently, three-dimensional (3D) displays have garnered interest because they offer realistic imagery in home entertainment, medical applications and computer-aided design applications $[1,2,3]$. Among the many types of 3D displays, an optical trickery-based system (i.e., binocular parallax) is most common, but it often causes simulation sickness, headache and nausea because the observers see different views in their right and left eyes and reconstruct a 3D image in their brains [3].

To solve this problem, volume displays that show $3 \mathrm{D}$ images in a $3 \mathrm{D}$ scene using volume-filling indicating elements have attracted significant interest [3]. Figure 1 shows the schematic view of the volume displays. A voxel is a cubic display element and a unit of a 3D image that is similar to a pixel in two-dimensional displays. Voxels are arrayed not only horizontally but also vertically and switch in 3D space to show an image. Thus, volume displays can prevent observers from feeling visual fatigue. However, transparent display elements must be arranged horizontally and vertically to see voxels inside displays, but few transparent display elements exist [3]. In addition, it is difficult to manufacture voxel structures at the hundreds of micrometer scale [4], whose size is determined by the resolution of human eyes, with conventional planer micro-fabrication processes.

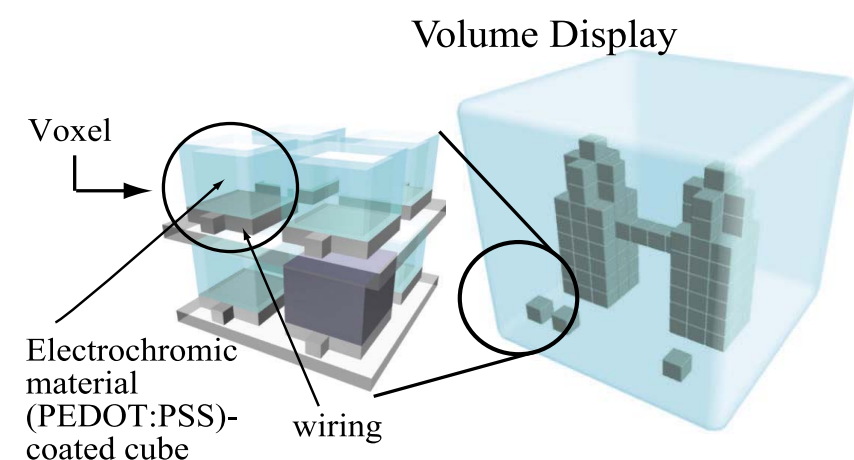

Fig. 1. Schematic view of volume display. Volume display consists of an electrochromic voxel array consists of electrochromic material-coated cubes and wirings.

To overcome these technical issues, we utilized organic-electrochromic display elements made of poly-(3,4-ethylenedioxythiophene):polystyrenesulfonate (PEDOT:PSS) as a transparent element. PEDOT:PSS electrically changes its color from transparent to blue according to the electrochromic effect [5]. To produce voxel elements, after cubic structures were constructed with ultraviolet (UV) curable polymer on a substrate through the MEMS process and coated with PEDOT:PSS, the resulting substrates were stacked in 3D space (Fig. 1). Previous studies have reported volume display devices that project images on a rotating screen [3], in air [6] and in water [7] with a scanning laser light. Compared with these systems, the PEDOT:PSS- 
based system will have advantages of expressing 3D images with 3D display elements and reducing the flickering of the scanning light. In this study, a fabrication process based on MEMS is described. The electrochromic element is evaluated, and the voxel operation is demonstrated.

\section{Structure and fabrication of PEDOT:PSS-based voxel array}

Figure 2 (a) shows the fabrication and structure of the proposed PEDOT:PSS-based voxel array. Two-dimensional voxel arrays are first fabricated and then stacked vertically. The voxel arrays are placed in an electrolyte with a counter electrode. If potentials are applied to selected voxels, the voxels change their color through a reversible electrochemical reaction in PEDOT:PSS.

(a)
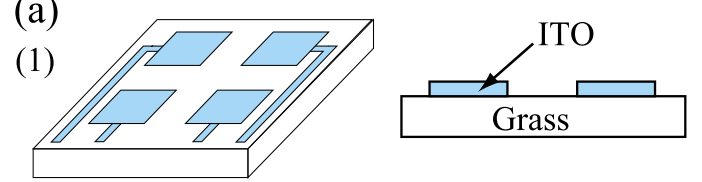

(2)
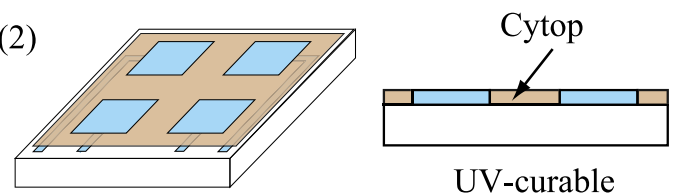

(3)

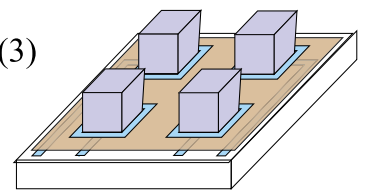

UV-curable

(4)
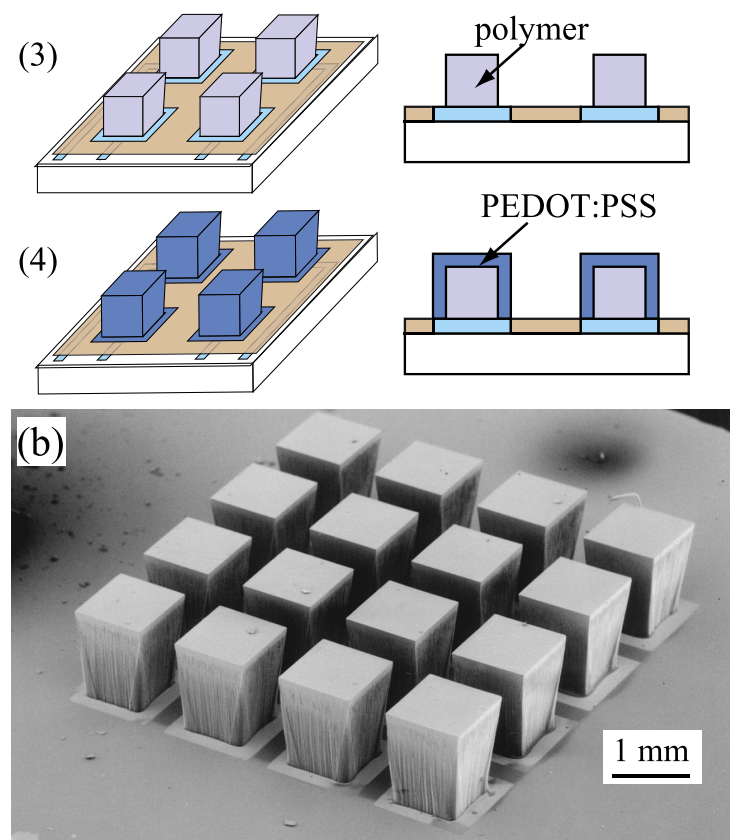

Fig. 2. (a) Fabrication process. (b) SEM image of fabricated voxel array.

The detailed process is described below.

(1) Fabrication starts by patterning a transparent electrode of Indium Tin Oxide (ITO) on glass. 
DOT:PSS, is coated and patterned by photolithography and oxygen plasma etching.

(3) A UV curable polymer (NOA65 Norland) which is not conductive is poured onto the resulting substrate to a thickness of $1 \mathrm{~mm}$ and exposed to UV light through a photo-mask. The UV polymer is developed by acetone and rinsed with ethanol.

(4) The fabricated substrate is dipped in a PEDOT:PSS solution and spun on a spinner. On the substrate, PEDOT:PSS coats the UV polymer structures because Cytop repels hydrophilic PEDOT:PSS solution. The PEDOT:PSS is dried at $100^{\circ} \mathrm{C}$ for one hour.

The fabricated voxel array is shown as an SEM image in Fig. 2 (b). The voxel is $1 \mathrm{~mm}$, and its pitch is $1.5 \mathrm{~mm}$, which is with in the range of conventional pixels (i.e., from 250 to $1000 \mu \mathrm{m}$ ) [4].

\section{Characterization of PEDOT:PSS electrochromism and oper- ation of voxels}

To characterize the electrochromism of PEDOT:PSS on an ITO electrode, a test film on ITO and a counter electrode of aluminum wire were placed in $100 \mathrm{mM} \mathrm{CaCl} 2$ solution, and different potentials were applied. Figure 3 (a) shows the light absorption spectra of the color changes with voltage of $-1.0 \mathrm{~V}$. Peak absorption was observed with a wavelength of $620 \mathrm{~nm}$; the absorption ratio changed from 0.1 to 0.5 while the voltage changed from $0 \mathrm{~V}$ to $-1.0 \mathrm{~V}$. Because this absorption wavelength is in the red region, the PEDOT:PSS film exhibits blue. When the voltage of $1.0 \mathrm{~V}$ was applied to the PEDOT:PSS, PEDOT:PSS changes its color from blue to transparent. The reversible electrochemical response of PEDOT:PSS was operated at the frequency of $1 \mathrm{~Hz}$.

Next, the fabricated voxel array was demonstrated to exhibit electrochromism. First, a two-dimensional array was soaked in a $100 \mathrm{mM}$ $\mathrm{CaCl}_{2}$ solution, and $-1.0 \mathrm{~V}$ was applied. One counter electrode of aluminum wire was placed at the edge of the voxel array and connected with all voxels in contact with electrolyte. The results are shown in Fig. 3(b). We observe the color change of the selected voxel, not only in the top view but also in the side view, which shows that the device can express voxels in $3 \mathrm{D}$ space. Therefore, our fabrication process could be utilized to construct a voxel array. Secondly, $3 \times 3 \times 3$ voxels were demonstrated. Three substrates of a two-dimensional array were stacked vertically and soaked in solution. The selected three voxels were operated by applying a potential of $-1.0 \mathrm{~V}$. Three voxels changed to blue, as shown in Fig. 3 (c). The coloration is pale because the light transmission rate of the PEDOT:PSS film caused by its non-uniformity and coloration efficiency is low. Solving these problems is the target of our future work to realize a $3 \mathrm{D}$ display.

\section{Conclusion}

We fabricated and demonstrated a volume display prototype using a 


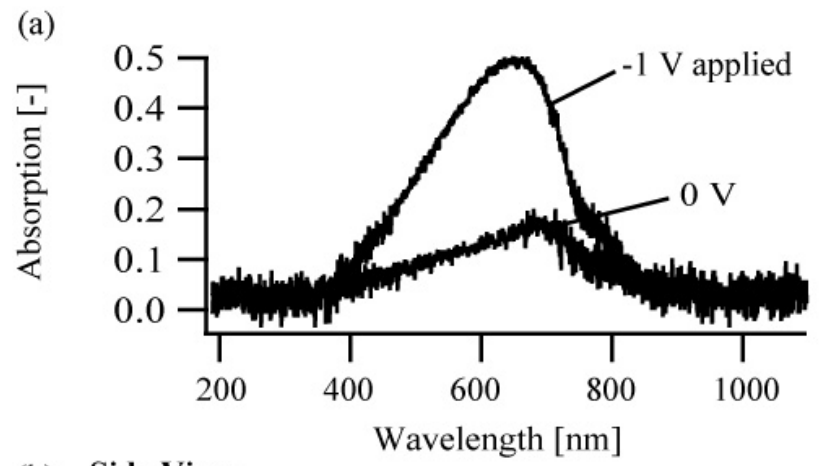

(b) Side View
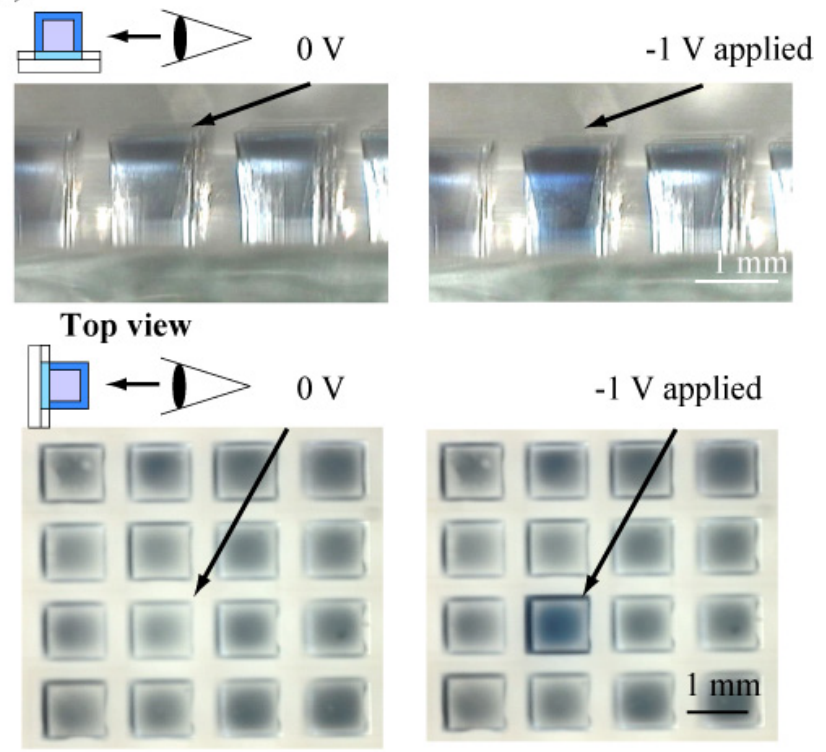

$-1 \mathrm{~V}$ applied

(c)

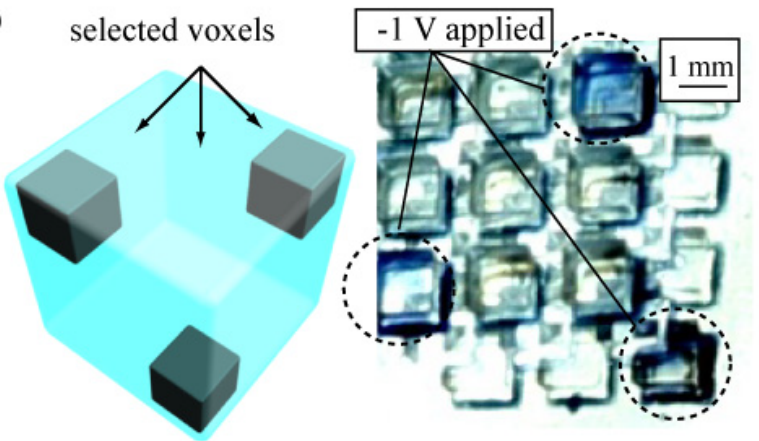

Fig. 3. (a) Absorption change of PEDOT:PSS. (b) Side view and top view of PEDOT:PSS voxel array. (c) Operation of $3 \times 3 \times 3$ voxels.

PEDOT:PSS-coated voxel array. PEDOT:PSS-electrochromism was characterized, and the peak of absorption was at a wavelength of approximately $620 \mathrm{~nm}$, where the absorption ratio changed from 0.1 to 0.5 under the applied potential of $-1.0 \mathrm{~V}$. The fabricated voxel array exhibits coloration that can be observed from the top and side views. Finally, a $3 \times 3 \times 3$ voxel array was assembled, and three selected voxels were changed from transparent to blue. Our proposed structure and fabrication can generate a volume display that consists of 3D voxel elements. 